\begin{tabular}{|c|c|c|c|c|c|}
\hline MUNIBE Antropologia-Arkeologia & $n^{\circ} 69$ & $63-73$ & DONOSTIA & 2018 & ISSN 1132-2217 • eISSN 2172-4555 \\
\hline
\end{tabular}

\title{
Cuentas de madera magdalenienses de la cueva de Santa Catalina (Lekeitio, Bizkaia, España)
}

\author{
Magdalenian wooden beads from the cave of Santa Catalina \\ (Lekeitio, Bizkaia, Spain)
}

\author{
PALABRAS CLAVES: Objetos de adorno. Cuentas de madera. Paleolítico Superior. Experimentación. \\ GAKO-HITZAK: Apaingarriak. Egurrezko aleak. Goi Paleolitoa. Esperimentazioa. \\ KEY WORDS: Decorative objects. Wooden beads. Upper Paleolithic. Experimentation
}

\section{Eduardo BERGANZA(1), Mónica RUIZ-ALONSO(2) y Rosa RUIZ IDARRAGA ${ }^{(3)}$}

\section{RESUMEN}

En las excavaciones realizadas en el yacimiento de Santa Catalina se han recuperado dos cuentas de madera de pequeño tamaño, forma circular y perforación central. Fueron encontradas en el nivel III atribuido al Magdaleniense superior.

Las piezas muestran signos de haber estado expuestas a una fuente de calor intenso por lo que presentan una importante termoalteración que ha facilitado su conservación.

Las cuentas han sido analizadas macro y microscópicamente lo que ha permitido reconocer la materia prima, aunque no concretar el tipo de madera seleccionada, y la forma en que se realizó la extracción.

Mediante un programa experimental hemos logrado reproducir el modo de fabricación y la manera en que pudieron haber sido ser utilizadas.

\section{LABURPENA}

Santa Catalina aztarnategian egindako indusketan, lepokoaren egurrezko ale bi aurkitu ziren Goi Madeleine aldiari dagokion III. mailan. Erreta egon direlako iraun izan dute gaur egun arte.

Aleok aztertu eta behatu egin dira mikroskopiarekin ere. Ezin jakin izan da zein egur motadunak diren, baina bai nola ebaki zen egurra Esperimentazioaren bitartez errepikatu egin da fabrikatzeko modua izan zitekeena eta nola erabili izan ziren.

\section{ABSTRACT}

The site of Santa Catalina is located in the coastal area of the Bay of Biscay, in Northern Spain, $35 \mathrm{~m}$ above present sea level.

Archaeological diggings performed in the site have shown a late glacial stratigraphical sequence that extends from the final Magdalenian to the Azilian period.

In level III, final Magdalenian (15.000 - 12.000 cal BP), we have been able to confirm a densely populated settlement, devoted to the exploitation of both sea and land resources. Within the collection of portable art recovered, there are two wooden beads, which are the subject of the present study.

These wooden beads are round pieces, of about $5 \mathrm{~mm}$ in diameter and with a central hole drilling. Both pieces are burnt, a fact which has contributed to their preservation.

The beads have been macro and microscopically analyzed, what has enabled us to determine its raw material, though the specific taxon has not been established. What we confirm is that both come from a tangential split of the wood.

Through an experimental survey, we were able to determine how these beads were manufactured and how they were used.

We have also resorted to other experimental studies which deal with the manufacturing of similar pieces from different materials.

From very small wooden bead blanks, of a size slightly larger than the beads and through the use of very fine micro drills, most likely hefted, the perforation must have resulted.

The final polish could have been produced by rubbing with some sandstone of fine grain

The small grooves observed in the holes suggest that these objects were hung, being part of a necklace or a pendant.

\section{INTRODUCCIÓN}

La cueva de Santa Catalina está enclavada en la costa cantábrica, al norte de la Península Ibérica. Sus dos bocas se abren sobre un acantilado a $35 \mathrm{~m}$ sobre el nivel actual del mar (Fig.1). Entre los años 1982 y 2000 se llevaron a cabo trabajos de excavación en una de sus bocas que se extendieron a un área máxima de $9 \mathrm{~m}^{2}$.

\footnotetext{
(1) AOZTA eduardoberganza@irakasle.eus

(2) Grupo de Investigación Arqueobiología, Instituto de Historia, CCHS, CSIC, C/ Albasanz, 26-28, 28037 Madrid. Monica.ruiz@cchs.csic.es

(3) rosa.ruizidarraga@gmail.com
} 


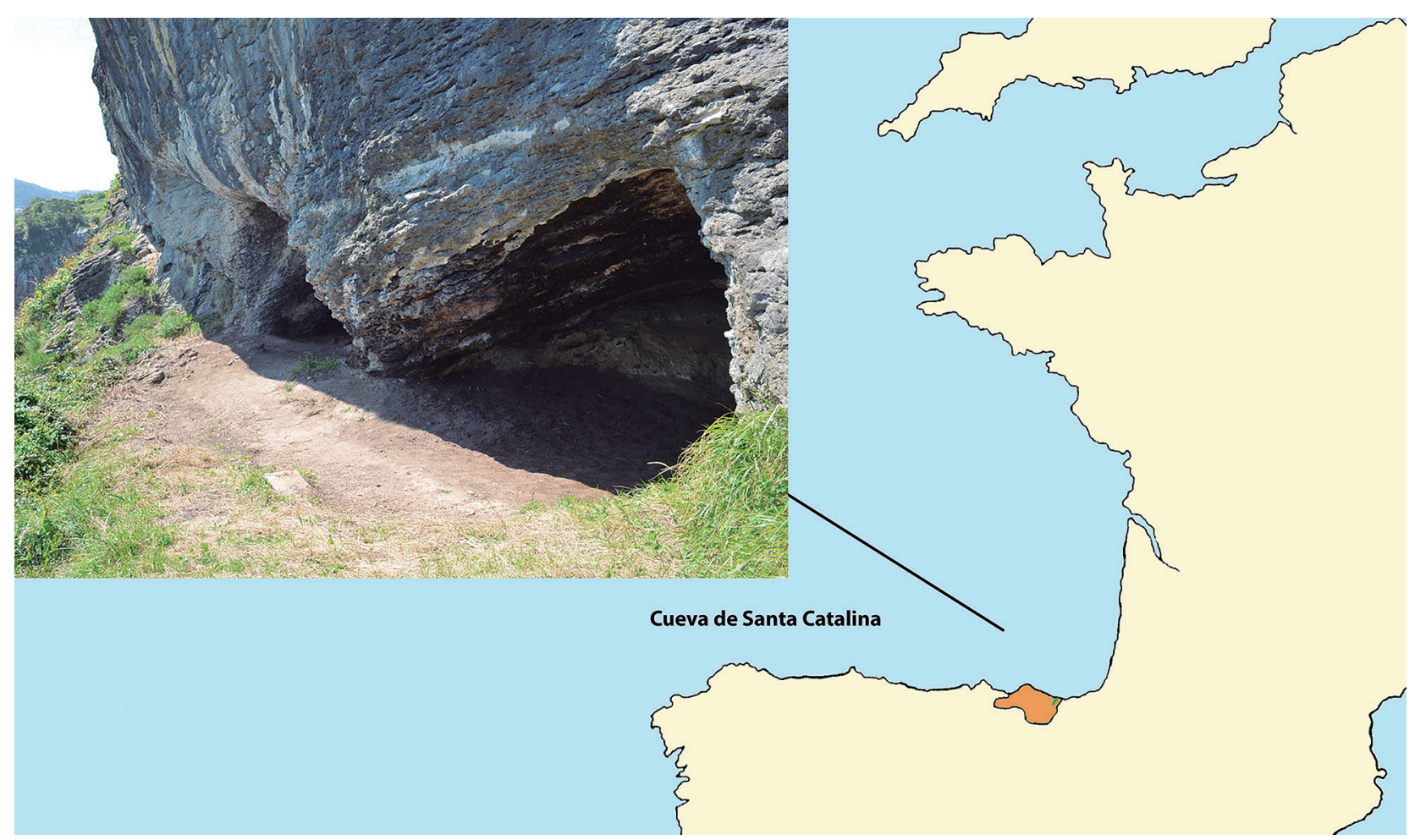

Fig. 1. Situación y boca de la cueva de Santa Catalina (Foto ADES) / Location and entrance to the Cave of Santa Catalina (Photo ADES).

En el relleno, de $150 \mathrm{~m}$ de espesor medio, se han documentado tres niveles cronoculturales que ponen de manifiesto una ocupación continuada e intensa del asentamiento entre el Magdaleniense Superior y el Aziliense (Berganza Gochi y Arribas Pastor, 2014a).

Para el cribado del sedimento se utilizó agua a presión y mallas de diferentes anchuras, siendo la menor de $0,5 \mathrm{~mm}$ de luz. Esto ha permitido una minuciosa recogida de todo tipo de evidencias y ha facilitado la recuperación de piezas de un tamaño muy reducido, como las que aquí se presentan, que de otro modo habrían pasado desapercibidas (Berganza Gochi y Arribas Pastor, 2014a).

Entre los materiales que componen el registro arqueológico de la cueva de Santa Catalina se recuperaron numerosos elementos de adorno elaborados sobre diversos soportes. Los objetos que analizamos en este trabajo son dos pequeñas cuentas realizadas en madera, que están bien conservadas. Se trata de un hallazgo excepcional dado que son muy escasas las piezas elaboradas en este material que se hayan recuperado en yacimientos paleolíticos debido a sus características perecederas.

Presentamos una descripción detallada de estas piezas, un análisis del soporte sobre el que están realizadas y una aproximación al reconocimiento de su fabricación y uso.

Fueron halladas en la parte inferior del nivel III que corresponde a un Magdaleniense superior. Ambas se encontraban en cuadros próximos y a una profundidad similar, con una diferencia de unos veinte centímetros.
Los datos concernientes a este nivel III indican que entre el 15.000 y el 12.000 cal BP se sucedieron ocupaciones en un entorno que se caracterizó por un ambiente paleoclimático frío aunque con oscilaciones algo más templadas (Berganza Gochi y Arribas Pastor, 2014c).

El paisaje estaría dominado por espacios abiertos junto a zonas boscosas más o menos desarrolladas. El análisis de los restos antracológicos de la madera utilizada como combustible, lleva a pensar que la cubierta vegetal incluiría algunos tipos de coníferas y abundantes arbustos (Ruiz-Alonso et al., 2014).

Una de las actividades más importantes que singularizaron este enclave, distante unos $5 \mathrm{~km}$ de la línea de costa durante el Magdaleniense superior, fue la explotación de los recursos del mar siendo habitual la pesca y la captura tanto de aves como de diversas especies de fauna marina. (Roselló Izquierdo y Morales Muñiz, 2014; Roselló-Izquierdo et al., 2016; Elorza Espelosin, 2014; Laroulandie, 2014; Laroulandie et al., 2016; Castaños Ugarte, 2014).

El utillaje de este nivel III está caracterizado por una industria ósea de arpones de una y dos filas de dientes y un equipamiento lítico de buriles, raspadores y abundantes laminillas y puntas de dorso. Destacan en este contexto las piezas de arte mueble y objetos de adorno (Berganza Gochi y Arribas Pastor, 2010; Berganza y Ruiz Idarraga, 2002, 2004 y 2014; Berganza et al., 2012; Ruiz Idarraga y Berganza, 2012). 


\section{METODOLOGÍA DE ESTUDIO}

Para determinar el tipo de material utilizado en la fabricación de estas piezas, así como para reconocer las técnicas empleadas en su fabricación, hemos realizado su observación con una lupa binocular estereoscópica Olympus SZX10 en el Arkeologi Museoa de Bilbao y con un Microscopio electrónico de barrido (MEB) de presión variable Hitachi 3400n, Type II, localizado en el Laboratorio de Microscopía Electrónica y Microanálisis (Microlab) del Instituto de Historia en el Centro de Ciencias Humanas y Sociales del CSIC de Madrid.

La dificultad en la utilización del MEB radicaba en la imposibilidad de realizar algunos protocolos básicos para la observación de materias vegetales como la metalización del material, debido a lo singular de las piezas. Por esta razón se introdujeron en el MEB sin metalizar y con una observación en bajo vacío. De igual manera, no podían fijarse los materiales a ninguno de los portamuestras del microscopio, como sí se hace con el resto de macrorrestos vegetales en el protocolo habitual. Por este motivo se preparó, para su estudio y fotografiado, un soporte especial de forma que no sufrieran ningún tipo de movimiento y así no se dañasen las piezas. Finalmente, y tras diversas variaciones del método utilizado, se decidió realizar una "cama" en aluminio bien ajustada para que la cuenta estuviera sujeta pero no pegada, dada la fragilidad del material, y que no interfiriera en la observación.

De forma preventiva se evitó que las piezas estuvieran durante un largo periodo de tiempo dentro del microscopio ya que se verían expuestas al haz de electrones, algo que podría dañarlas. Por este motivo, una vez analizadas las cuentas en el microscopio electrónico, se realizaron diferentes micrografías para certificar lo observado y poder continuar con su estudio.

Hemos llevado a cabo un programa experimental con objeto de tratar de conocer el proceso de fabricación de las cuentas replicando tanto la forma como la perforación y el acabado. Igualmente hemos observado cuentas de madera pertenecientes a collares modernos a fin de reconocer los estigmas que el uso pudiera haber dejado en ellas.

\section{RESULTADOS}

\subsection{Morfología}

\subsubsection{Cuenta A (SC.A8.L31C.5205)}

La pieza tiene forma discoidea con una perforación central de delineación circular. Su diámetro es de 4,4 $\mathrm{mm}$ y su grosor de 2,1 mm. El diámetro de la perforación es de 1,9 mm (Fig.2).

El contorno exterior forma un ángulo recto con las paredes y tiene unas aristas exteriores ligeramente redondeadas.
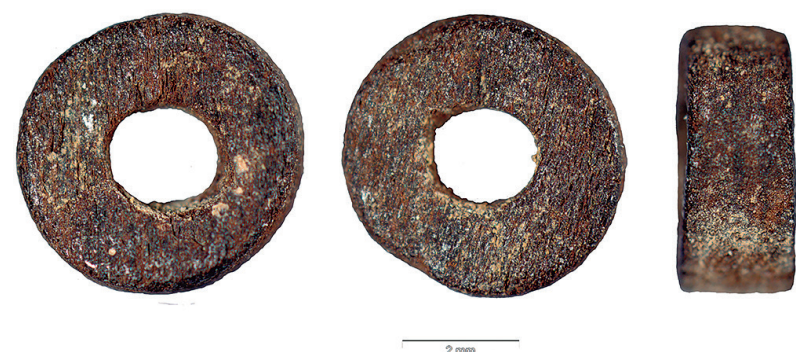

Fig. 2. Cuenta A (SC.A8.L31C.5205) / Bead A (SC.A8.L31C.5205).

La perforación central presenta aristas marcadas y unas paredes casi verticales en su mayor parte. Sin embargo, hay una zona, que se repite en ambas caras, en la que se produce un biselado de la arista haciendo que la pared sea en parte oblicua.

En una de las caras de la perforación hay una muesca de forma abiselada que afecta tanto a la superficie de la cuenta como a parte de la pared del orificio. En esa misma arista hay una melladura irregular y pequeñas muescas de dimensiones reducidas (Fig.3). En la otra cara únicamente se observan muescas de este último tipo.

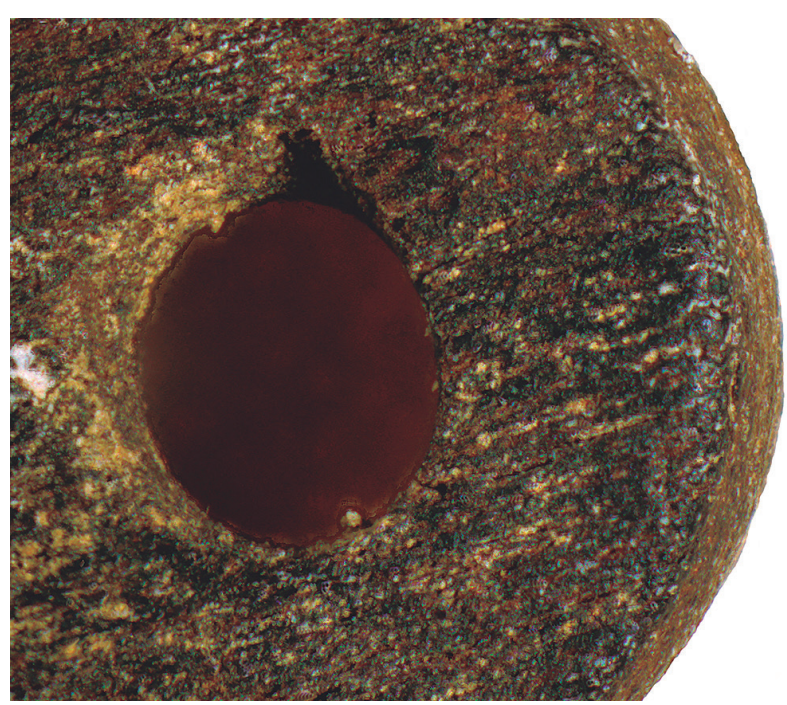

Fig. 3. Detalle de la muesca en la perforación de la cuenta A / Detail of the groove on the perforation of bead $A$

Es difícil la observación detallada del interior de la perforación. Sin embargo, se aprecia que tiene una sección interior bicónica.

El contorno exterior es muy regular y no presenta ninguna alteración o rebaje en su grosor. La superficie de ambas caras, anverso y reverso, están alisadas hasta el punto de hacer imposible reconocer los poros o fibras naturales del material.

La cuenta presenta un buen estado de conservación, a excepción de una pequeña saltadura de materia en una de las caras y ligeras erosiones en la otra. Tiene 
un color marrón bastante uniforme que es consecuencia de un proceso de termoalteración, así como pequeños puntos de color negro brillante que corresponden a zonas precarbonizadas. Se observan algunas adherencias calizas postdeposicionales que cubren zonas muy reducidas que no impiden el análisis del objeto.

No se observa en el exterior ningún tipo de sustancia adherida que haga pensar en un tratamiento de abrasión con un material colorante añadido. Tampoco la existencia de un recubrimiento de la superficie natural que haya servido para tintar el objeto.

\subsubsection{Cuenta B (SC.B8.L31F.1135)}

La forma de esta pieza es circular, ligeramente ovalada. Sus dimensiones son 5,27 por 5,40 mm y una altura de 3,04 mm. Al igual que la cuenta anterior, presenta una perforación redonda en su centro cuyo diámetro es de 1,5 mm (Fig.4).
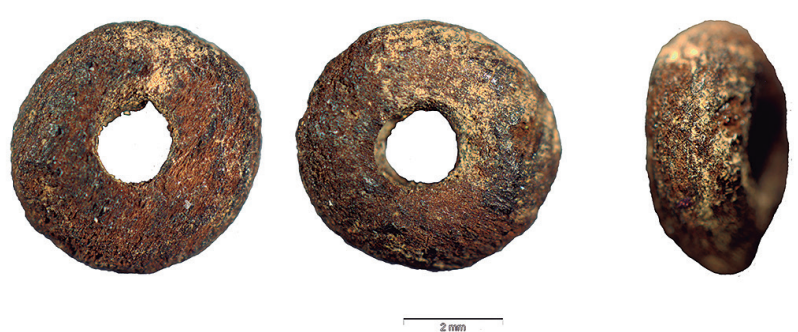

Fig. 4. Cuenta B (SC.B8.L31F.1135) / Bead B (SC.B8.L31F.1135).

El aspecto general es semejante al de la otra cuenta, aunque las aristas del contorno son algo más redondeadas. Éstas son muy regulares y no se observan muescas en ellas. No hay diferencia en su espesor con respecto al resto de la cuenta.

La perforación central muestra una arista aguda en la mayor parte de su contorno y algunas zonas en las que está redondeada o rebajada.

En ambas caras observamos la existencia de dos amplias muescas abiseladas que afectan a parte del orificio y se corresponden entre sí. Junto a ellas existen pequeñas muescas y alguna melladura todas de carácter irregular (Fig. 5)

Su parecido con la otra pieza también se hace evidente en el canal de la perforación en el que se observa un resalte en su parte central que marca una sección bicónica.

También en este caso, tanto en las caras como en el contorno, se aprecia un acabado que ha regularizado la superficie eliminando los poros de la madera. Por esta razón se hace difícil distinguir la fibra original.

El estado de conservación es bueno aunque se observan algunas saltaduras superficiales y una erosión algo más importante, con pequeñas pérdidas de
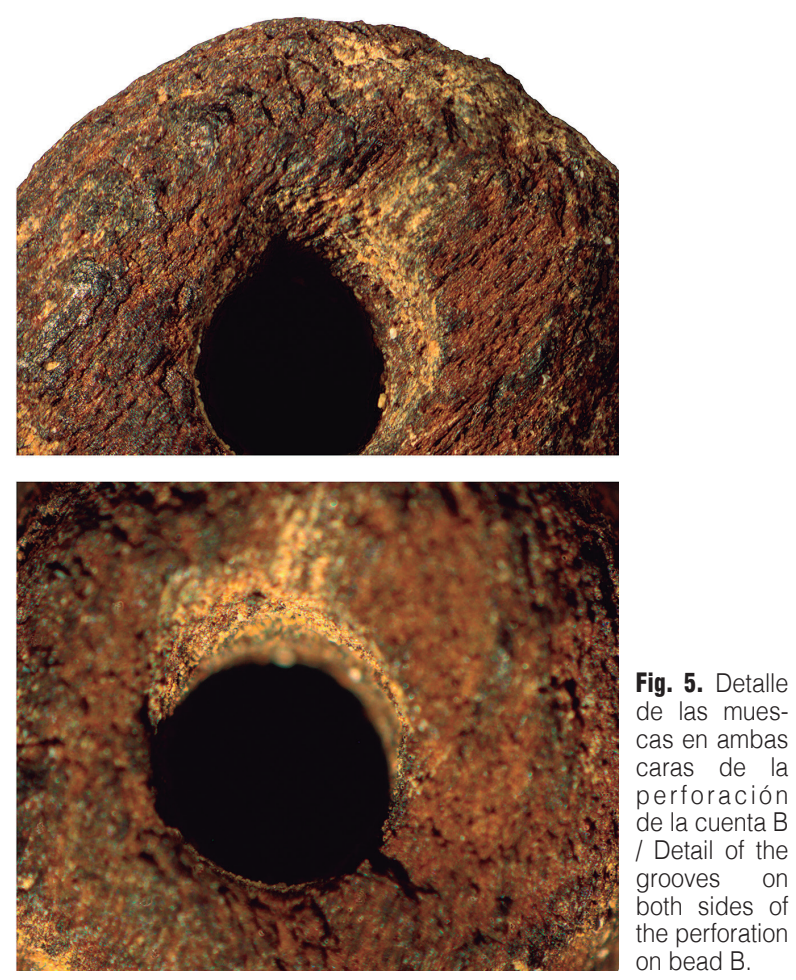

materia. La cuenta está totalmente termoalterada como se deduce del color marrón uniforme. Presenta zonas puntuales que han sufrido una afectación térmica mayor y que han llegado a carbonizarse. Tiene, además, algunas concreciones calizas sobre la superficie.

Tampoco hemos observado en este ejemplar rastros superficiales que denoten algún tipo de tratamiento, de abrasión o tintado.

La forma discoidea con una perforación central es frecuente entre las piezas hechas sobre concha, piedra, hueso u otros materiales y puede considerarse un modelo estandarizado (Álvarez Fernández, 2006; Stiner, 2014; Taborin, 1993). Las piezas de Santa Catalina responden a este tipo y no presentan ninguna novedad formal que las haga diferentes.

\subsection{La materia prima}

La identificación taxonómica de la madera se basa en la determinación anatómica de las tres secciones que presenta su estructura y su comparación con las actuales (Fig. 6). Se trata de una técnica sencilla que no necesita ningún proceso químico para su manipulación. En el caso que nos ocupa existen limitaciones que no han permitido la identificación taxonómica debido a que no se han podido definir características anatómicas diagnósticas. Dado la excepcionalidad de estas piezas no hemos podido recurrir, como así se hace cuando las secciones a examinar se encuentran ocultas por la suciedad, pulido, etc., a la fragmentación con 
SECCIÓN TRANSVERSAL

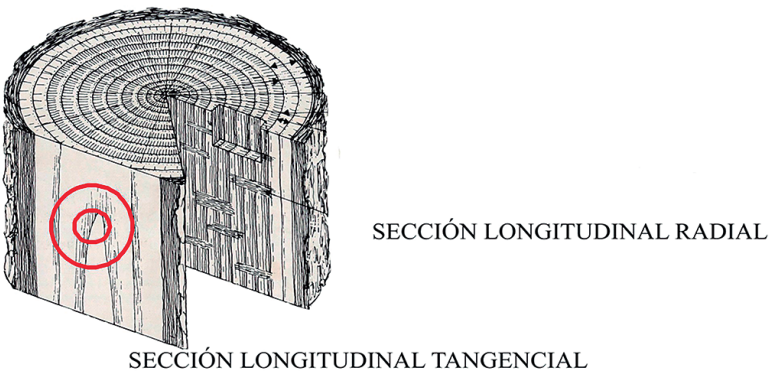

Fig. 6. Representación de las secciones macroscópicas de la madera (redibujado de Schweingruber, 1990: 14) / Design of the macroscopic tangential splits on the wood (adapted from Schweingruber, 1990: 14). la mano, orientando cada fractura hacia los tres planos anatómicos mencionados lo que permite de manera más o menos rápida y bastante fiable la determinación concreta de muchos de los taxones.

Sí se observaron algunas estructuras parciales que certificaban que el material sobre el que se habían realizado era madera, aunque no pudimos establecer un diagnóstico preciso sobre el taxón empleado. Como se puede ver en las figuras 7 y 8 , en la sección imagen mostrada, se observan fibras correspondientes a una sección longitudinal radial, viéndose aquí posiblemente las paredes de las células aunque sin que se pueda identificar claramente su estructura.

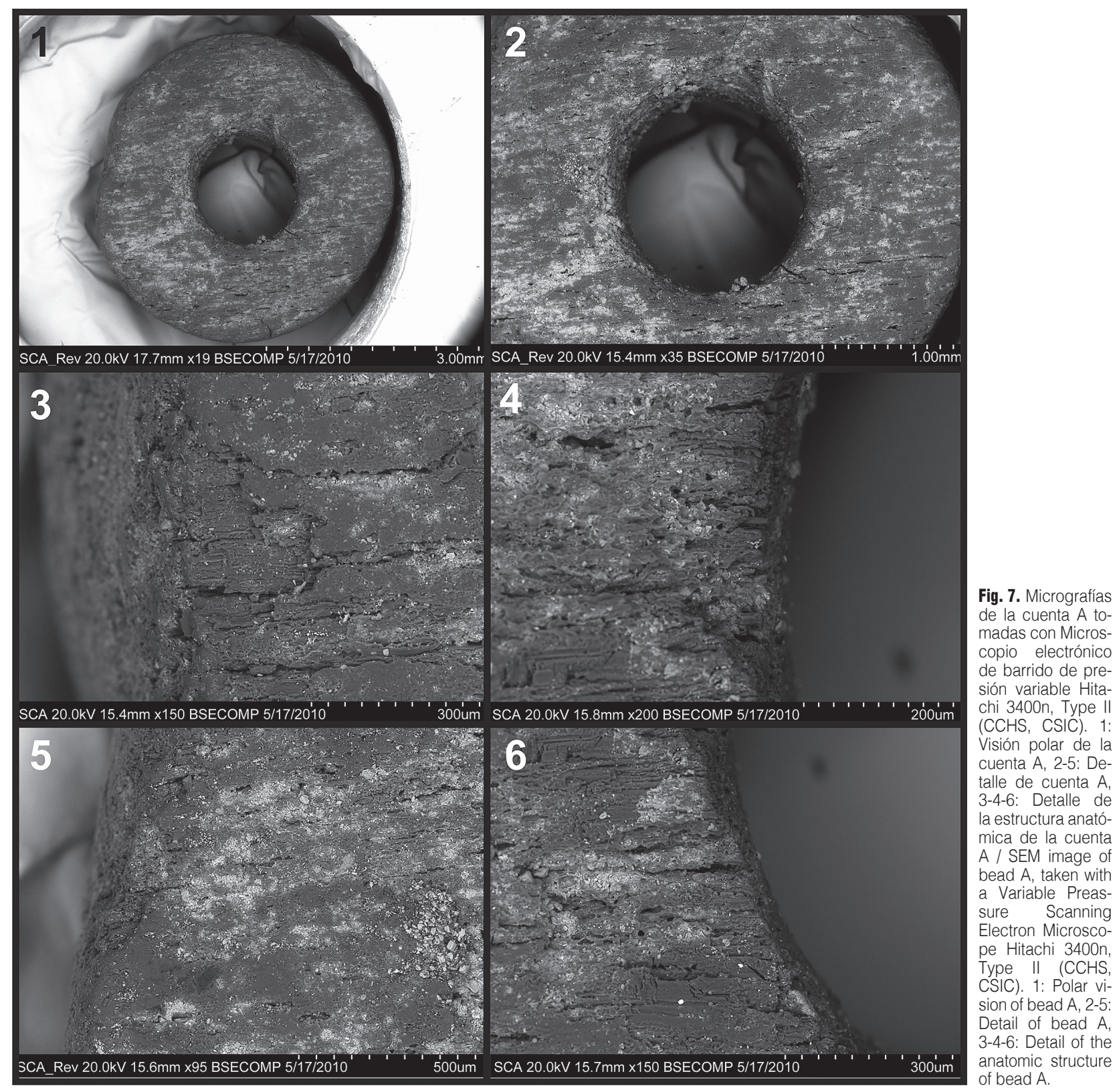




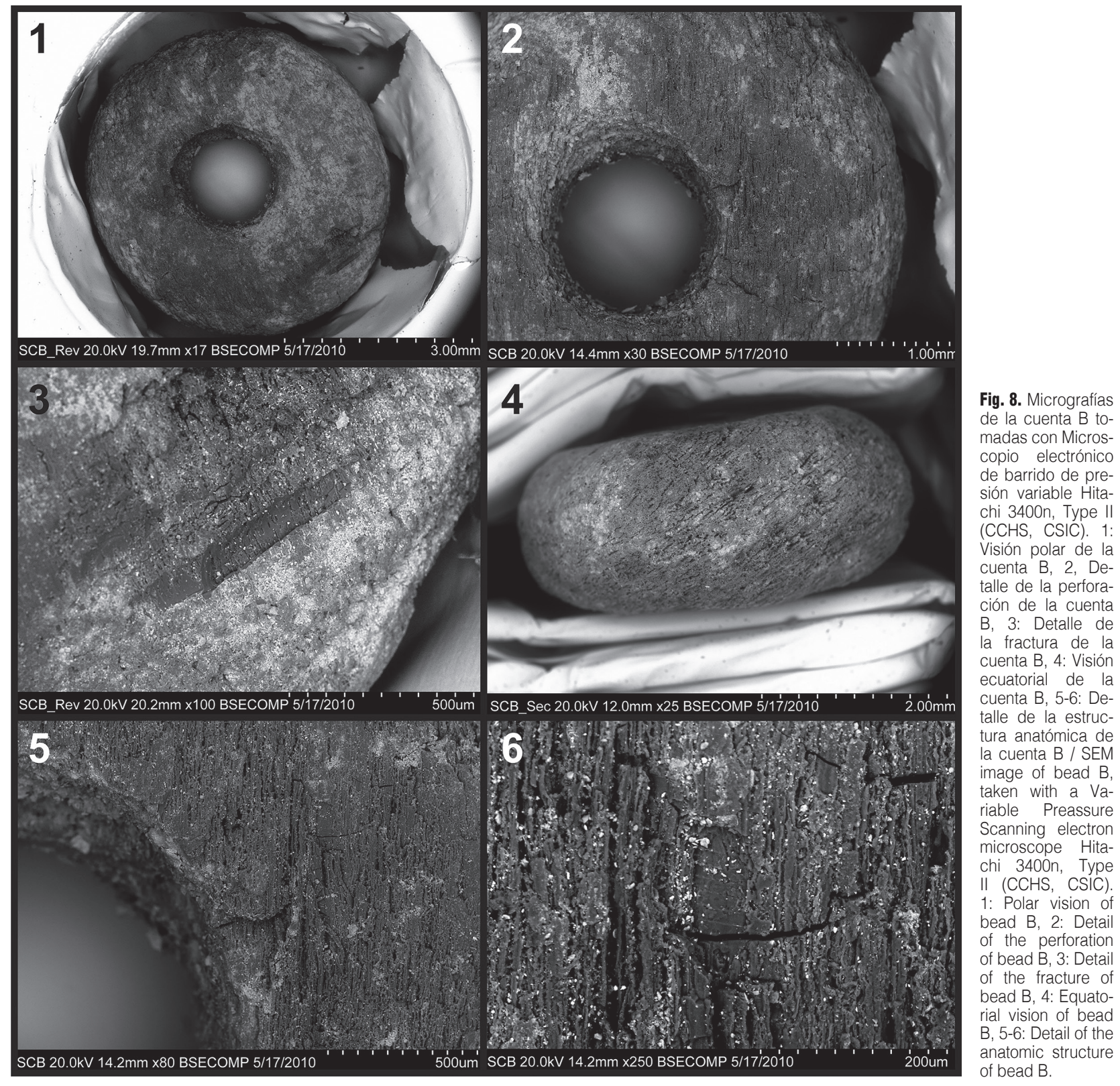

En la observación microscópica de las cuentas hemos podido apuntar la dirección de las fibras, lo que permite reconocer cómo estarían realizadas. Así, en la visión polar de las cuentas, si seguimos la sección en la que está realizada la perforación, vemos la dirección de las paredes de las células por lo que tendría un formato que atravesaría la sección longitudinal radial (Fig. 7, 8 y 9). En su visión ecuatorial se observa la terminación de estas fibras, por lo que podríamos apuntar a que se trata de la sección transversal de la madera. Hemos situado la imagen de una de las cuentas, a modo de esquema, sobre la representación de las secciones macroscópicas de la madera publicado en el trabajo de Schweingruber (1990: 14) (ver detalles en Fig. 6).
Los recursos vegetales debieron tener un papel muy importante en las estrategias de supervivencia de los grupos cazadores recolectores. Así lo evidencian, entre otros elementos, las figuraciones, tanto en el arte parietal como en el mobiliar, que se han interpretado como representaciones de plantas y que nos mostrarían una explotación habitual de este recurso (Tyldesley y Bahn, 1983).

En los análisis de huellas de uso en los útiles de sílex recuperados en la cueva de Santa Catalina, se confirma que durante el Magdaleniense se ha trabajado la madera en el asentamiento. Sin embargo, como es habitual en las etapas finales del Paleolítico superior, ese uso tiene una escasa presencia en relación al realizado sobre otros materiales (Ibáñez Estévez y González Urquijo, 1996). 


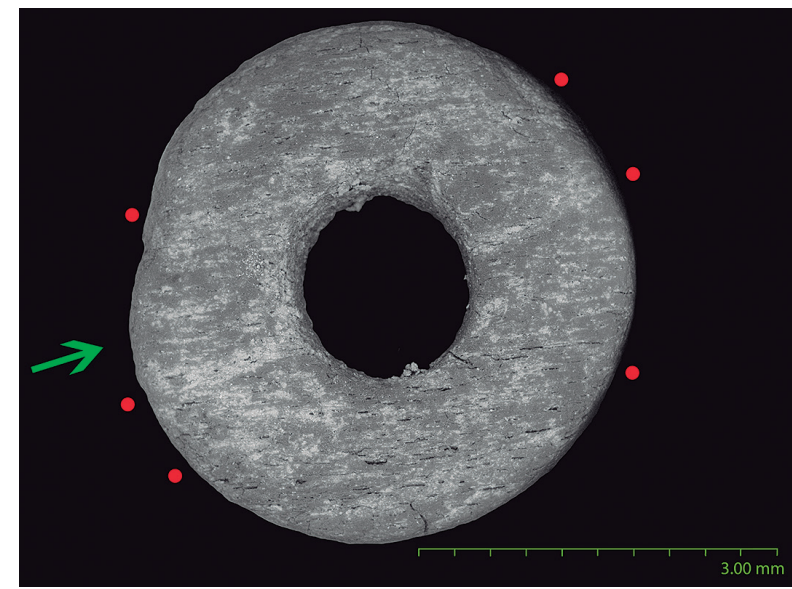

Fig. 9. Vista de la cuenta B en la que se marca la dirección de las paredes de la sección radial, marcando así la dirección en la que se ha realizado la cuenta / View of bead B, where the direction of the radial tangential split is marked, and thus, showing the direction in which the bead was obtained.

El empleo de tablas de corte tangencial en la fabricación de útiles prehistóricos es bastante habitual, tal y como ocurre en nuestras cuentas, y se ha señalado que pueda deberse a que este tipo de corte al no romper el grano de la madera tiene la ventaja de mantener la solidez y resistencia naturales de la madera (Coles et al., 1978).

\subsection{Técnicas de fabricación}

Puesto que no conocíamos el tipo de madera en el que estaban realizadas, recurrimos como material experimental a astillas de dos de los taxones arbóreos presentes en el nivel III (Ruiz-Alonso et al., 2014). Hemos utilizado el pino y el roble con objeto de contrastar materiales de dureza diferente.

En primer lugar, obtuvimos unas tablillas alargadas, de forma rectangular y poco grosor, aunque más espesas que las cuentas arqueológicas. Estas tablillas procedían de soportes obtenidos de un corte tangencial al hilo de las fibras de la madera, de acuerdo con lo constatado en la observación de las piezas originales.

En nuestra experimentación probamos a preparar una tablilla con perforaciones dispuestas a una distancia regular para luego separar los fragmentos (Fig.10). Sin embargo, este método no resultaba adecuado puesto que se rompía muy a menudo. Por esta razón elegimos serrar fragmentos con una lasca de sílex y sobre ellos llevar a cabo la perforación (Fig.11). Esta labor no supuso ninguna dificultad.

Para la perforación del orificio central empleamos la punta de un perforador y también un triedro de sílex. El trabajo producía la rotura o el embotamiento frecuente del extremo del útil por lo que para mantener una punta activa aguda había que reavivarlo reiteradamente.

La horadación se realizó desde ambas caras del fragmento de madera obteniendo un orificio bicónico cuyo perfil era algo rebajado. También en estos casos

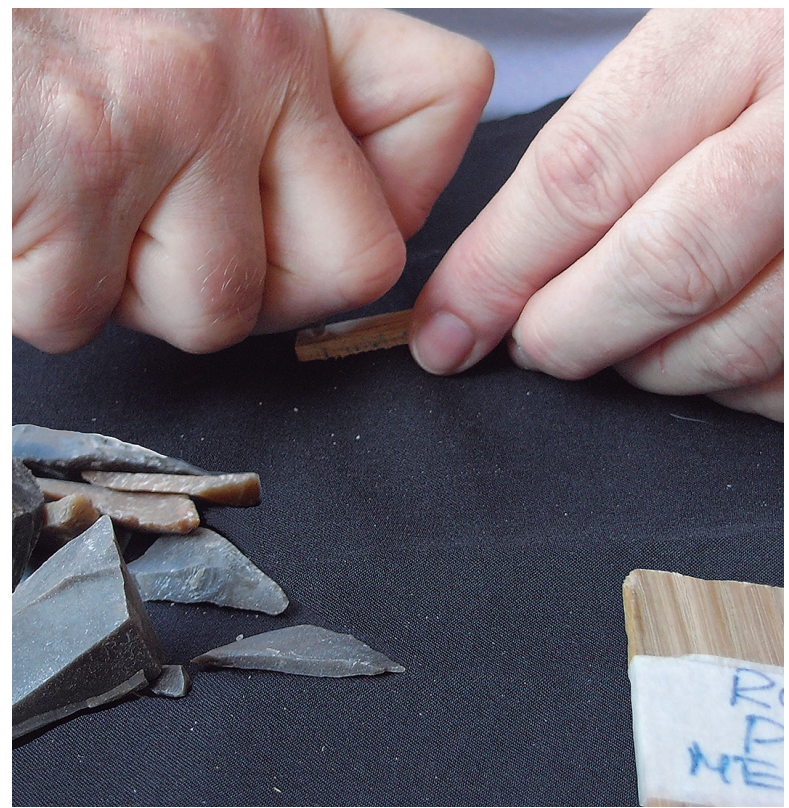

Fig. 10. Experimentación. Perforación por medio de un buril de sílex / Experimentation. Hole drilling with a flint burin.

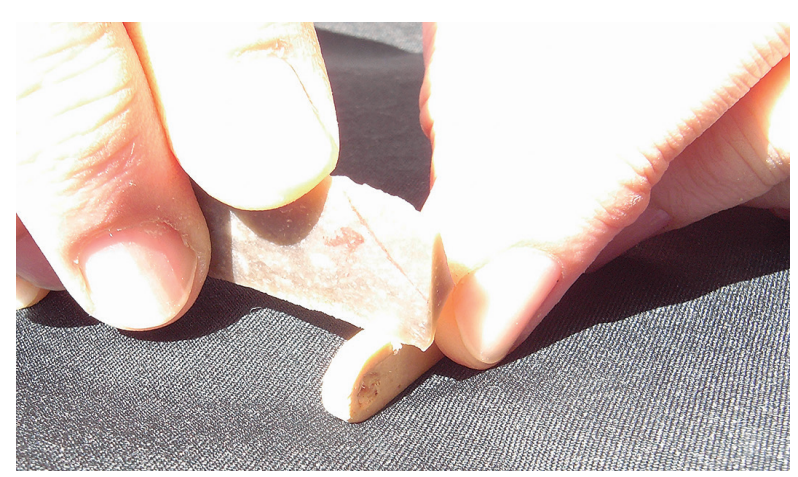

Fig. 11. Experimentación. Corte de una tablilla con una lámina de sílex / Experimentation. Cut of a small plank with a flint blade.

se producía el astillado y la fractura del soporte lo que nos hizo concluir que el método que estábamos empleando no era el idóneo.

Únicamente hemos encontrado mención a hallazgos magdalenienses de cuentas realizadas en madera en la cueva de Las Caldas (Asturias,España). En las publicaciones se indica el hallazgo de tres cuentas hechas en esta materia orgánica. Las piezas se recuperaron en la sala II. Dos de ellas corresponden al Magdaleniense medio (CAL.-056 y CAL.-064) y la tercera a un Magdaleniense medio/superior (CAL.-040). En ninguno de los ejemplares se precisa el tipo de madera con el que se hicieron, su forma, su estado de conservación o las técnicas empleadas en su fabricación lo cual nos ha impedido tener una referencia de piezas contemporáneas que pudieran asemejarse a las nuestras y con las que hubiéramos podido establecer una comparación (Corchón, 1992; Álvarez Fernández, 2006). 
En la bibliografía existente relativa a la fabricación de cuentas perforadas de tamaño similar a estas que presentamos, aunque en materias primas distintas, se citan diversas estrategias experimentales (Yerkes, 1993; Wright et al., 2008).

En el estudio de pequeñas cuentas circulares en piedra recuperadas en yacimientos neolíticos del Próximo Oriente se ha constatado el empleo de perforadores de sílex mucho más estrechos que los habituales y de puntas activas hechas con recortes de buril, tanto de ángulo como sobre truncadura. El propio retoque de los recortes de los buriles sobre truncadura crea una sección prismática que permite producir, mediante una perforación eficiente y rápida, orificios netos al tiempo que se reduce el número de las fracturas (Wright et al., 2008).

Las piezas de sílex necesarias para esta función son de tamaño tan reducido que es imposible su manejo directamente con las manos, por lo que se hace necesario su enmangado en algún ástil, posiblemente de madera.

El rozamiento constante del giro regular de la punta, más que la presión que se ejerce con ella, es el que produce la horadación. El trabajo se hace desde ambas caras y en algunas ocasiones se procede a perforar por segunda vez para regularizar el interior. Los orificios que se obtienen son pequeños y con aristas más regulares que las que habíamos conseguido nosotros.

En el sudeste de los Estados Unidos de América también se ha podido constatar el trabajo de perforación de cuentas de concha por medio de perforadores sujetos en un vástago, accionados con el mismo método empleado para la obtención del fuego, o por medio de sencillos taladros de arco (Yerkes, 1993).

No podemos desechar la hipótesis de que cualquiera de los métodos descritos pudo haber servido para la fabricación de las cuentas de Santa Catalina, aunque no hay referencias bibliográficas de su uso en el Magdaleniense europeo.

La conformación, el redondeamiento del contorno y la regularización de las superficies, es una labor sencilla recurriendo al uso de un canto de arenisca. Sin embargo, es difícil hacerlo manualmente de forma individual dadas las reducidas dimensiones de las cuentas. Además, esta dificultad en la manipulación impide conseguir la regularidad de la forma que tienen las cuentas, un orificio tan perfectamente centrado y unas aristas exteriores tan bien acabadas.

Algunos investigadores han hecho referencia a métodos de pulido de cuentas no de forma individual sino de manera conjunta, en especial cuando se trata de piezas de pequeñas dimensiones. En primer lugar se procede a su ensartado por medio de un hilo y, después, el conjunto se sujeta sobre un soporte y se frota con areniscas que actúan como elemento activo en la acción de desgaste (Yerkes, 1993; Ricou y Esnard, 2000) (Fig. 12).

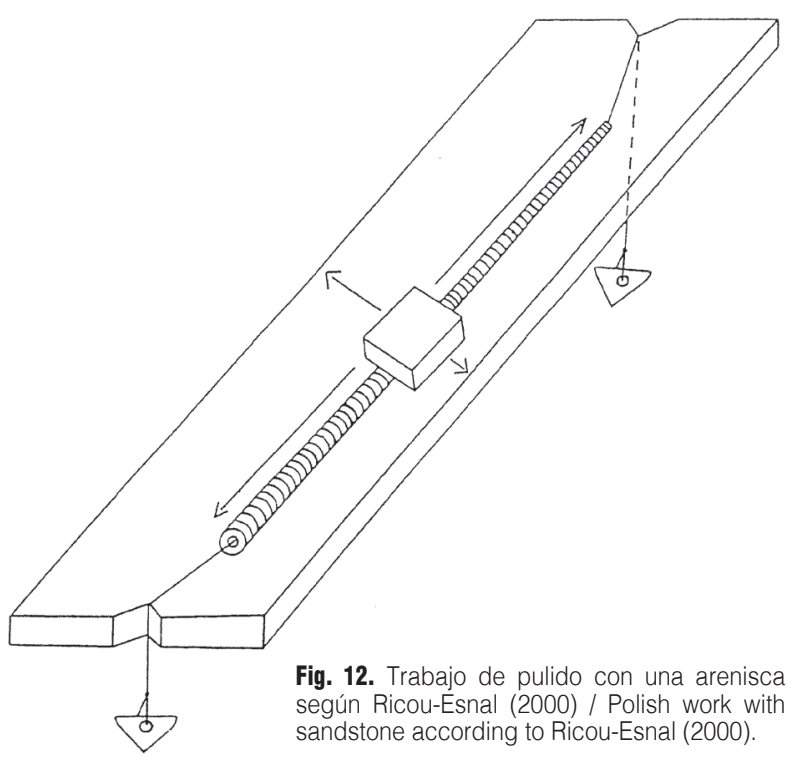

El estado de termoalteración tan parecido que presentan ambas cuentas suscita la cuestión de si se debe a una manipulación intencionada durante su fabricación o es consecuencia de un hecho fortuito postdeposicional. Algunos trabajos experimentales realizados para la obtención de cuentas en madera parece que llevan a la conclusión de que esta materia prima sometida al fuego se hace más frágil y comporta una mayor tendencia a las fracturas, por lo que su exposición a una fuente de calor resulta inadecuada como paso previo a su conformación y perforación (Oliva, 2015).

Sin embargo, sí se ha planteado la posibilidad de que las cuentas una vez conformadas hayan podido ser sometidas a la acción del fuego para darles una mayor durabilidad. En el enterramiento neolítico F.3667 de Catalhoyuk se recuperaron cuentas de madera semejantes a las que ahora estudiamos: de un tamaño similar, circulares, con perforación central y completamente quemadas. Como en el caso que nos ocupa, el acabado y alisado de las mismas ha hecho imposible la identificación de la madera sobre la que están hechas. Dado que aparecieron en un contexto en el que no hay otros restos de cremación, la hipótesis que plantearon fue que el contacto con un foco de calor intenso pudo ser intencionado buscando una mayor perdurabilidad del material o un acabado de color oscuro (Asouti y Kabukcu, e.p.; Asouti, 2013).

En este sentido queremos señalar que en los diferentes momentos de la ocupación del yacimiento de Santa Catalina se desarrollaron importantes y frecuentes actividades de combustión (Berganza Gochi y Arribas Pastor, 2014b). Como consecuencia, una parte significativa del material industrial así como de los restos faunísticos que se han recuperado conservan señales inequívocas de haber estado sometidos a fuentes de calor que han provocado en ellos termoalteraciones más o menos intensas. 
El estudio de las alteraciones que se produjeron en los restos óseos ha dejado claro que éstas han sido importantes en toda la secuencia estratigráfica, siendo más intensas en el nivel III. En este nivel llega a estar afectada la quinta parte de los restos óseos indeterminados y casi el $46 \%$ de los determinados. No se ha podido concluir de manera segura que este hecho se deba a una acción intencionada, resultado del uso de estos materiales como combustible, como se ha podido constatar en los yacimientos cantábricos de El Horno (Costamagno y Fano, 2004), Labekokoba (Yravedra et al., 2005) o en algunos localizados en territorio francés (Costamagno et al., 2009). Por el contrario, más bien parece que en una elevada proporción el origen de la termoalteración en estos restos pudo ser su proximidad a estructuras de combustión (Arribas y Berganza, 2010).

Es por ello que debemos considerar como muy probable la hipótesis de que el proceso de quemado de las cuentas, de manera similar al de otras evidencias de su mismo conjunto estratigráfico, pudo haberse producido de forma fortuita tras su abandono.

\subsection{USO}

Ambas cuentas presentan en la arista de la perforación unas pequeñas muescas biseladas. En las cuentas de madera modernas, que hemos utilizado ensartadas como collares, aparecen muescas similares provocadas por la erosión o incisión que ha producido su roce con el cordón (Fig. 13).

No hemos podido detectar muescas semejantes en el perímetro exterior de los objetos arqueológicos. Este hecho nos indica que no sufrieron en esa parte el roce de elemento alguno de sujeción por lo que redunda en la idea de que estuvieron suspendidas en un collar o en un colgante y que no estuvieron cosidas.

Considerando el reducido tamaño de cada una de las cuentas, así como el hecho de que ambas sean de un mismo material, tengan dimensiones y forma muy similares y que la técnica empleada en su fabricación sea semejante, nos inclinan a pensar que formaban parte de un mismo conjunto.

Las cuentas y otros objetos, tradicionalmente interpretados como adornos, entrarían dentro de los útiles con funciones simbólicas, culturales o sociales (Moro Abadía y Nowell, 2015). Una forma o un material determinado puede haber sido elegido como un rasgo identificativo, bien de un estatus bien de una condición distintiva (Kuhn, 2014; Stiner, 2014; Vanhaeren y D'Errico 2006; Whitte, 1995, 2007).

Podemos pensar que estos objetos poseían unas características visuales que los hacían fáciles de reconocer como pudo ser el color, el brillo, la dificultad en su fabricación, etc. (Stiner, 2014).

Otra forma de singularizarlas podría haber sido recurrir al tintado de las piezas para darles mayor vistosidad, pero como ya se ha indicado no hemos podido determinar ningún rastro de colorante. Hemos de tener en cuenta que la alteración por el fuego podría haber hecho desaparecer las posibles coloraciones.

La visibilidad podría lograrse con una gran cantidad de estas pequeñas piezas o con la combinación de varios elementos. Queremos resaltar que en Santa Catalina han sido recuperados varios colgantes en concha, piedra o hueso (Berganza et al., 2012; Ruiz Idarraga y Berganza, 2012) y elementos de adorno que podrían ir suspendidos o adheridos (Berganza Gochi y Arribas Pastor, 2010).
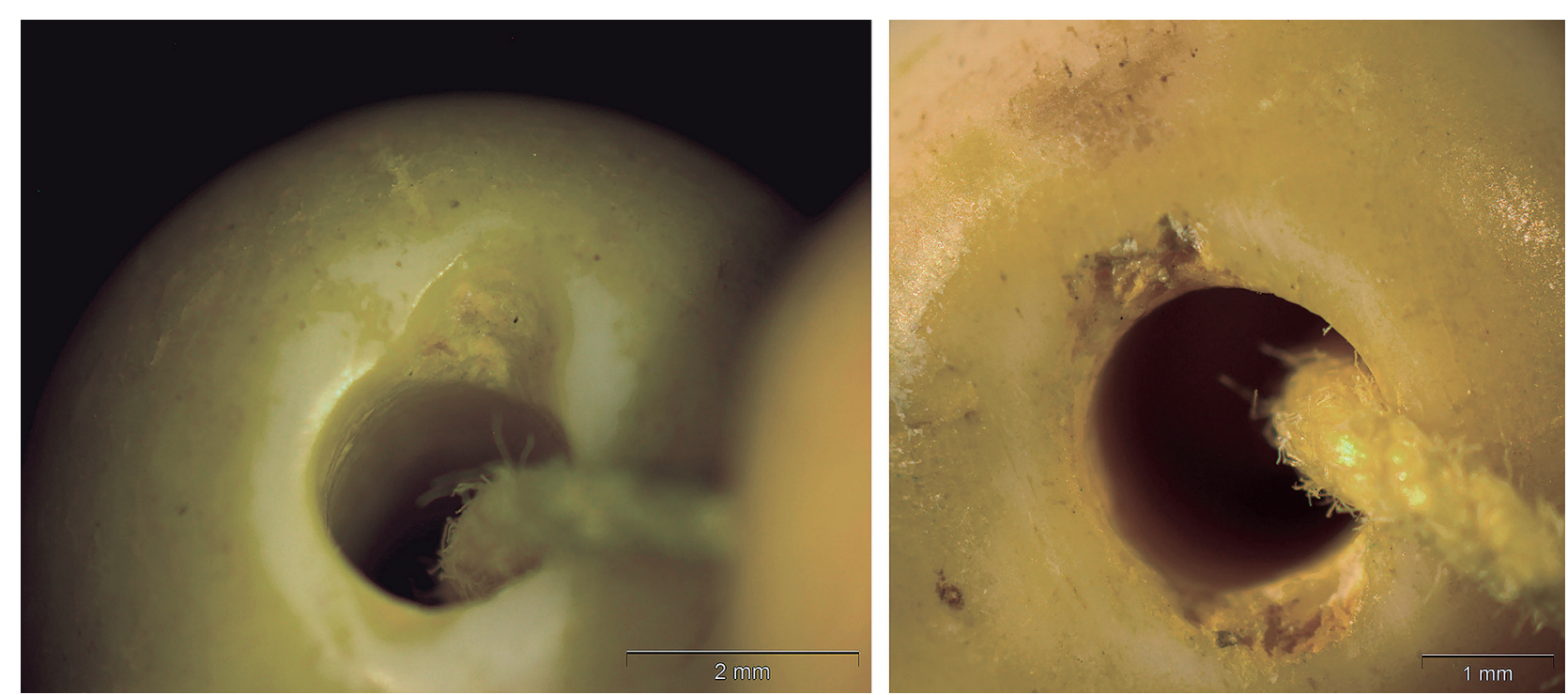

Fig. 13. Marcas producidas por el roce del hilo en cuentas de madera de collares modernos / Resulting marks from the rubbing of the thread on wooden beads of modern necklaces. 


\section{CONCLUSIONES}

Hemos podido identificar que el soporte empleado ha sido una varilla de madera obtenida por medio de un corte tangencial puesto que la posición de las fibras así lo indica. No hemos podido, sin embargo, conocer el taxón empleado.

Un programa experimental nos ha hecho ver como más probable que la fabricación en serie de estas cuentas partiría de unas tablillas o soportes finos y alargados. Estos soportes se dividirían por medio del serrado en fragmentos rectangulares, de unas dimensiones algo mayores a las de las cuentas.

La perforación se pudo conseguir empleando una punta activa fina con la que se actuaría desde los dos laterales. Este útil pequeño y estrecho incidiría en la madera de forma reiterada pero con poca presión, de manera que el orificio obtenido fuera de diámetro reducido y su arista no se redondearía en exceso.

El acabado o redondeamiento de las cuentas se llevaría a cabo por medio de frotación con una roca arenisca.

La comparación de los estigmas presentes en las piezas arqueológicas con los de cuentas de madera similares ensartadas en collares actuales nos muestra que es muy posible que se trate de piezas que se usaron suspendidas y no cosidas.

El hecho de que estén en un estado de precarbonización ha permitido su conservación. Creemos, sin embargo, que se debe a un caso fortuito y refleja la intensa actividad crematoria que se ha desarrollado en el asentamiento.

\section{AGRADECIMIENTOS}

Lydia Zapata fue la primera persona que certificó la naturaleza vegetal de la materia prima y nos animó en la investigación de la misma. Iñaki Libano y Jesús E. González Urquijo nos han proporcionado amablemente materias primas y útiles líticos empleados en la experimentación. Mónica Ruiz-Alonso está financiada por el Programa Estatal de Promoción del Talento y su Empleabilidad en I+D+i en la modalidad Juan de la Cierva.

\section{BIBLIOGRAFÍA}

Álvarez Fernández, E., 2006. Los objetos de adorno-colgantes del Paleolítico superior y del Mesolítico en la Cornisa Cantábrica y en el valle del Ebro: una visión europea. Universidad de Salamanca, Salamanca.

Arribas, J. L., Berganza, E., 2010. Los restos óseos quemados del yacimiento tardiglaciar de la cueva de Santa Catalina (Lekeitio, Bizkaia). Estudio preliminar. Kobie 29, 19-34.

Asouti, E., 2013. Woodland vegetation, firewood management and woodcrafts at Neolithic Catalhöyük. In: Hodder, I. (Ed.), Humans and landscapes of Catalhöyük. Reports from 20002008 seasons, 129-161. British Institut at Ankara, London.
Asouti, E., Kabukcu, C., Vegetation and woodland management history. Worked wood. In: Tung, Burcu, CATALHOYUK. Archive report 2012, 238-246. Available from: http://archeo. edu.pl/catal/catal2012.pdf.

Berganza Gochi, E., Arribas Pastor, J. L., 2010. Dientes de herbívoros serrados e incisos de la cueva de Santa Catalina (Lekeitio, Bizkaia). Munibe Antropologia-Arkeologia 61, 57-70.

Berganza Gochi, E., Arribas Pastor, J. L., 2014a. La cueva de Santa Catalina (Lekeitio, Bizkaia): La intervención arqueológica. Restos vegetales, animales y humanos. BAI 4. Diputación Foral de Bizkaia, Bilbao.

Berganza Gochi, E., Arribas Pastor, J. L., 2014b. Estructuras de combustión. In: Berganza Gochi, E., Arribas Pastor, J. L. (Coords.), La cueva de Santa Catalina (Lekeitio, Bizkaia): La intervención arqueológica. Restos vegetales, animales y humanos, 33-48. Diputación Foral de Bizkaia, Bilbao.

Berganza Gochi, E., Arribas Pastor, J. L., 2014c. El entorno físico de las ocupaciones de Santa Catalina. In: Berganza Gochi, E., Arribas Pastor, J. L. (Coords.), La cueva de Santa Catalina (Lekeitio, Bizkaia): La intervención arqueológica. Restos vegetales, animales y humanos, 367-378. Diputación Foral de Bizkaia. Bilbao.

Berganza, E., Arribas, J. L., Ruiz Idarraga, R., 2012. Estudio tecnológico de los moluscos marinos perforados de los yacimientos de Lumentxa y Santa Catalina (Lekeitio, Bizkaia). Munibe Antropologia-Arkeologia 6, 93-104.

Berganza, E., Ruiz Idarraga, R., 2002. Un colgante decorado magdaleniense del yacimiento de Santa Catalina (Lekeitio, Bizkaia). Munibe Antropologia-Arkeologia 54, 67-77.

Berganza, E., Ruiz Idarraga, R., 2004. Una piedra, un mundo. Un percutor magdaleniense decorado. Diputación Foral de Álava, Vitoria-Gasteiz.

Berganza, E., Ruiz Idarraga, R., 2014. Candil de ciervo decorado del yacimiento de Santa Catalina (Bizkaia, España). Tecnología y funcionalidad. Munibe Antropologia-Arkeologia 65, 25-36.

Castaños Ugarte, P., 2014. Estudio de los macromamíferos del yacimiento de Santa Catalina. In: Berganza Gochi, E., Arribas Pastor, J. L. (Coords.), La cueva de Santa Catalina (Lekeitio, Bizkaia): La intervención arqueológica. Restos vegetales, animales y humanos, 331-360. Diputación Foral de Bizkaia, Bilbao.

Coles, J. M., Heal, S.V.E., Orme, B. J., 1978. The use and character of wood in prehistoric Britain and Ireland. Proceedings of the Prehistoric Society 44, 1-45.

Corchón, M. S., 1992. La cueva de Las Caldas (Priorio, Oviedo). II Investigaciones efectuadas entre 1987 y 1990. Excavaciones arqueológicas en Asturias 1987-90, 33-47. Servicio de Publicaciones del Principado de Asturias, Oviedo.

Costamagno, S., Fano, M. A., 2004. Practiques cynégétiques et exploitation des ressources animales dans les niveaux du magdalénien supérieur-final de El Horno (Ramales, Cantabrie, Espagne). Paleo 17, 31-56.

Costamagno, S., Théry-Parisot, I., Castel, J.-CH., Brugal, J-PH., 2009. Combustible ou non? Analyse multifactorielle et modèles explicatifs sur des ossements brûles paléolithiques. In: Théry-Parisot, I., Costamagno, S., Henry, A. (Ed.), Gestion des combustibles au paléolithique et au mésolithique. Nouveaux outils, nouvelles interpretations, 65-84. Actes du XVème Congrès UISPP, Lisboa 2006. Oxford: BAR International Series 1914, Oxford. 
Elorza Espelosin, M., 2014. Explotación de aves marinas en el Tardiglacial del golfo de Bizkaia: Las aves de Santa Catalina. In: Berganza Gochi, E., Arribas Pastor, J. L. (Coords.), La cueva de Santa Catalina (Lekeitio, Bizkaia): La intervención arqueológica. Restos vegetales, animales y humanos, 263-296. Diputación Foral de Bizkaia, Bilbao.

Ibáñez Estévez, J. J., González Urquijo, J. E., 1996. From tool use to site function. Use-wear analysis in some Final Upper Palaeolithic sites in the Basque country. BAR international series, Oxford.

Kuhn, S. L., 2014. Signaling theory and technologies of communication in the Paleolithic. Biological Theory 9, 42-50.

Laroulandie, V. 2014. Traitement et utilisation des ressources aviaires au Tardiglaciaire dans la grotte de Santa Catalina. In: Berganza Gochi, E., Arribas Pastor, J. L. (Coords.), La cueva de Santa Catalina (Lekeitio, Bizkaia): La intervención arqueológica. Restos vegetales, animales y humanos, 297-330. Diputación Foral de Bizkaia, Bilbao.

Laroulandie, V., Elorza Espolosin, M., Berganza Gochi, E., 2016. Les oiseaux marins du Magdalénien supérieur de Santa Catalina (Lekeitio, Biscaye, Espagne). Approches taphonomiques et archéozoologique. In: Dupont, C., Marchand D., Archéologie des chasseurs-cueilleurs maritimes. De la fonction des habitats à l'organisation de l'espace littoral. S.P.F., Paris.

Moro Abadía, O., Nowell, A., 2015. Palaeolithic personal ornaments: Historical development and epistemological challenges. Journal of Archaeological Method and Theory 22, 952-979.

Oliva Poveda, M., 2015. Aprofitament I transformació de matèries primeres per a l'elaboració d'ornaments durant la prehistòria recent (5600-3400 cal. ane) al nord-est de la península ibérica. Universitat Autónoma de Barcelona, Barcelona.

Ricau, CH., Esnard, TH., 2000. Étude experimentale concernant la fabrication de perles en coquillage de deux sites artenaciens oléronais. B.S.P.F. 97, 83-93.

Roselló-Izquierdo, E., Berganza-Gochi, E., Nores-Quesada, C., Morales-Muñiz, A., 2016. Santa Catalina (Lequeitio, Basque Country): An ecological and cultural insight into the nature of prehistoric fishing in Cantabrian Spain. Journal of Archaeological Science: Reports 6, 645-653

Roselló-Izquierdo, E., Morales-Muñiz, A., 2014. Las ictiofaunas de Santa Catalina (Lequeitio, Vizcaya): Un registro singular para la prehistoria cantábrica. In: Berganza Gochi, E., Arribas Pastor, J. L. (Coords.), La cueva de Santa Catalina (Lekeitio, Bizkaia): La intervención arqueológica. Restos vegetales, animales y humanos, 161-262. Diputación Foral de Bizkaia, Bilbao.

Ruiz-Alonso, M., Uzquiano Ollero, P., Zapata Peña, L., 2014. Macrorrestos vegetales de Santa Catalina (Lekeitio, Bizkaia): carbones y bellotas del Tardiglaciar. In: Berganza Gochi, E., Arribas Pastor, J. L. (Coords.), La cueva de Santa Catalina (Lekeitio, Bizkaia): La intervención arqueológica. Restos vegetales, animales y humanos, 75-92. Diputación Foral de Bizkaia, Bilbao.

Ruiz Idarraga, R., Berganza, E., 2012. Un colgante magdaleniense de la cueva de Santa Catalina. Análisis tecnológico y funcional. Isturitz 12, 61-81.

Stiner, M.C., 2014. Finding a common bandwidth: Causes of conver gence and diversity in paleolithic beads. Biological Theory 9, 51-64.

Schweingruber, F. H., 1990. Microscopic Wood Anatomy. Swiss Federal Institute for Forest, Snow and Landscape Research.
Tyldesley, J. A., Bahn, P. O.,1983. Use of plants in the european Palaeolithic: A review of the evidence. Quaternary Science Reviews 2, 53-81.

Taborin, Y., 1993. La parure en coquillage au Paléolithique. CNRS, Paris.

Vanhaeren, M., D'Errico, F., 2006. Aurignacien ethno-linguistic geography of Europe revealed by personal ornaments. Journal of Archaeological Science 33, 1105-1128.

White, R., 1995. Ivory personal ornaments of Aurignacian age: technological, social and symbolic perspectives. In: Hahn, J., Menu, M., Walter, PH., Widemann, F., Le travail et l'usage de l'ivoire au Paléolithique supérieur. Ravello 26-31 mai 1992, 2962. Istituto Poligrafico e Zecca dello Stato, Roma.

White, R., 2007. Systems of personal ornamentation in the Early Upper Palaeolithic: Methodological challenges and new observations. In: Mellars, P., Boyle, K., Bar-Yosef, O., Stringer C., Rethinking the human revolution new behavioural and biological perspectives on the origin and dispersal of modern humans, 287-302. Universidad de Cambridge, Cambridge.

Wright, K. I., Critchley, P., Garrard, A., Baird, D., Bains, R., Groom, S., 2008. Stone bead technologies and early craft specialization: insights from two Neolithic sites in Eastern Jordan. Levant 40(2), 131-165.

Yerkes, R. W., 1993. Methods of manufacturing shell beads at prehistoric Mississippian sites in southeastern North America. In: Anderson, P. C., Beyries, S., Otte, M., Plisson, H., Traces et function: les gestes retrouvés. Eraul 50, 235-242.

Yravedra, J., Baena, J., Arrizabalaga, A., Iriarte, M. J., 2005. El empleo del material óseo como combustible durante el Paleolítico Medio y Superior en el Cantábrico. Observaciones experimentales. Actas de la reunión científica: Neandertales Cantábricos. Estado de la cuestión, 369-383. Monografías del Museo Nacional y Centro de Investigación de Altamira $n^{\circ} 20$. Madrid. 
